\title{
Evaluation of left ventricular global functions with speckle tracking echocardiography in patients recovered from COVID-19
}

\author{
Savaş Özer ${ }^{1}$ (i) $\cdot$ Lütfullah Candan $^{2} \cdot$ Ali Gökhan Özyıldız · Oğuzhan Ekrem Turan²
}

Received: 30 December 2020 / Accepted: 1 March 2021 / Published online: 16 March 2021

(c) The Author(s), under exclusive licence to Springer Nature B.V. 2021

\begin{abstract}
Highly sensitive troponin (hs-TnI) levels are frequently elevated in COVID-19 patients and are associated with increased cardiovascular mortality during hospitalization. However, no data exists on cardiac involvement in patients recovered from COVID-19 infection. We aimed to evaluate by global longitudinal strain (LV-GLS) whether there is subclinical myocardial deformation after COVID-19 infection. Two-dimensional speckle tracking echocardiography (2D-STE) was performed within $29.5 \pm 4.5$ days after COVID-19 treatment. The standard GLS limit was identified at $<-18 \%$. The patients were divided into two groups according to their hs-TnI levels during hospitalization as with $(>11.6 \mathrm{ng} / \mathrm{dl})$ and without $(<11.6 \mathrm{ng} / \mathrm{dl})$ myocardial injury. Patients' $(\mathrm{n}=74)$ mean age was 59.9 years, and women were in the majority $(60.8 \%)$. Of the patients, $43.2 \%$ of them were hypertensive, and 10.9\% were diabetic. Abnormal LV-GLS values $(>-18)$ were measured in 28 patients $(37.8 \%)$. While $16(57.1 \%)$ of these patients were in the group with myocardial injury, $12(26.1 \%)$ of them were in the group without myocardial injury $(\mathrm{p}=0.014)$. D-dimer, $\mathrm{C}$ reactive protein, white blood cell levels were higher in the group with myocardial injury (All $\mathrm{p}$ values $<0.05$ ). Electrocardiographically, $9(12.2 \%)$ patients had $\mathrm{T}$ wave inversion, while two patients had a bundle branch block. Subclinical left ventricular dysfunction was observed in approximately one-third of the patients at the one-month follow-up after COVID-19 infection. This rate was higher in those who develop myocardial injury during hospitalization. This result suggests that patients recovered from COVID-19 infection should be evaluated and followed in terms of cardiac involvement.
\end{abstract}

Keywords Coronavirus · COVID-19 infection · Left ventricular global longitudinal strain · Transthoracic echocardiography

\section{Introduction}

Coronavirus disease-2019 (COVID-19), develops due to coronavirus-2 (SARS-CoV-2), has caused a high morbidity and mortality secondary to its pandemic worldwide. Although the respiratory system is at the forefront, COVID19 can affect many systems, including the cardiovascular system [1,2]. COVID-19 infection can affect the myocardium directly or via cytokine storm [3-5]. In the infection's active period, various forms of myocardial injury have been

Savaş Özer

savasozer87@gmail.com

1 Trabzon Kanuni Training and Research Hospital Cardiology Clinic, Trabzon, Turkey

2 Faculty of Medicine Department of Cardiology, Karadeniz Technical University, Trabzon, Turkey

3 Recep Tayyip Erdogan University Training and Research Hospital Cardiology Clinic, Rize, Turkey reported, identified using cardiac troponin levels, transthoracic echocardiography (TTE), and cardiac magnetic resonance imaging [3-8]. Myocardial injury secondary to COVID-19 is associated with poor prognosis during hospitalization $[9,10]$. It is unclear whether myocardial injury that may develop during the infection is permanent in the short or long term. Cardiac MRI revealed that up to $90 \%$ of patients had myocardial edema or cardiac involvement after COVID-19 infection [7].

2D speckle tracking echocardiography (2D-STE) can diagnose subclinical myocardial dysfunction earlier than conventional echocardiography $[11,12]$. Various studies have reported an inverse correlation of increased troponin levels with global longitudinal strain (GLS) values in the population without cardiovascular disease [13]. Studies in the literature about the increase in cardiac troponin levels and cardiac involvement due to COVID-19 during hospitalization are present [3]. However, it should be explained whether cardiac involvement continues in long term after 
COVID-19 infection, the level of cardiac involvement, if any, and which patient group is more risky. At this point, it becomes important to determine which patient group should be follow-up and treated for long-term cardiac involvement. We aimed to perform electrocardiographic and echocardiographic evaluations at the first-month visit in patients recovered from COVID 19.

\section{Materials and method}

\section{Study population}

Patients hospitalized with the diagnosis of COVID-19 between September 27 and October 30, 2020, and admitted to the cardiology clinic for routine control, were included in the study. COVID-19 diagnosis was accepted as the positive polymerase chain reaction (PCR) test of the nasopharyngeal swab. Causes that may increase troponin levels or clinical conditions that may cause deterioration in LVGLS analysis were determined as exclusion criteria. Coronary artery disease (11 patients), heart failure (two patients), atrial fibrillation (six patients), previous cerebrovascular disease (two patients), renal failure (eGFR $<30 \mathrm{ml} / \mathrm{min} / 1.73^{\mathrm{m} 2}$ ) (nine patients), severe chronic obstructive pulmonary disease (COPD) (four patients), malignancy (one patient), poor echogenicity (18 patients), and being under 18 years old were the exclusion criteria (Fig. 1). After the exclusion criteria were applied, the study continued with 74 patients. Twodimensional echocardiography (2DE) images were obtained from the patients one month after discharge. The upper limit of the troponin test kit was $11.6 \mathrm{ng} / \mathrm{L}$ in our hospital laboratory. The cut off limit of CRP was accepted $5 \mathrm{mg} / \mathrm{l}$ and above. By taking the upper limit of troponin as reference, the

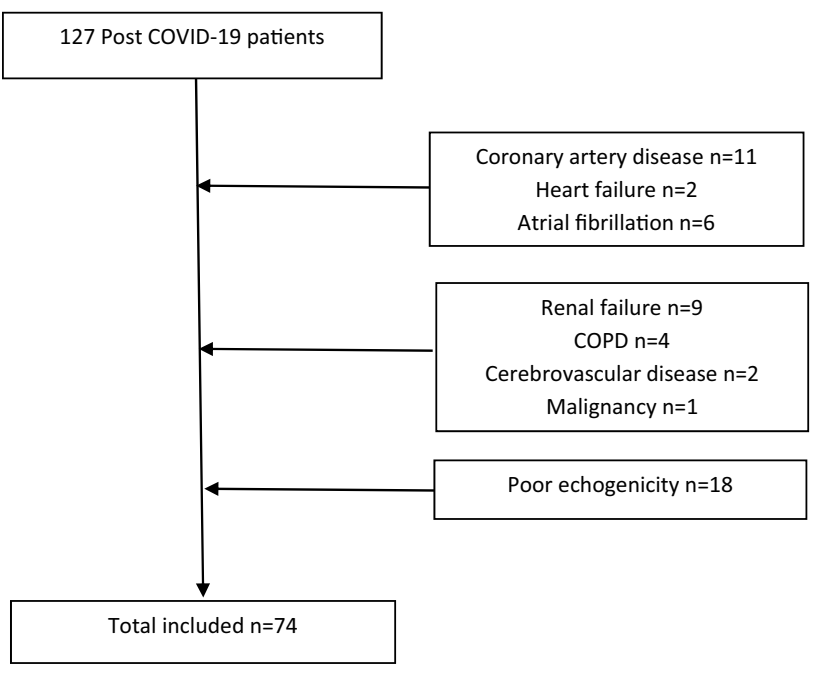

Fig. 1 Study flow chart, Post COVID-19 patients patients were divided into two groups according to their hsTnI levels at hospitalization. The study was conducted under the Helsinki Declaration's principles and was approved by the regional ethics committee (No: 2020-56). Informed consent was obtained from patients before the study.

\section{Obtaining demographic and laboratory data}

The patients' demographic characteristics and medical history were recorded in the first-month visit. The laboratory findings (glucose, complete blood count, renal function tests, C-reactive protein (CRP), hs-TnI, and D-dimer) were obtained by retrospective analysis of the biochemistry results studied during their hospitalization due to COVID-19.

\section{Echocardiography}

Echocardiographic examination (Philips Epiq7; Philips Healthcare, Inc., Andover, MA, ABD) was performed on patients one month after discharge. Echocardiographic images were obtained and recorded by standard techniques (parasternal long axis, short axis, apical two chambers, apical three chambers, and apical four chambers). Left ventricular end-systole and end-diastolic diameters, left atrium diameter, left ventricular posterior wall thickness, and interventricular septum thickness were measured according to the American Echocardiography Association guidelines [14]. Left ventricular ejection fraction (LVEF), left ventricular end-systolic volume (LV-ESV), and left ventricular enddiastolic volume (LV-EDV) were measured by the modified Simpson's rule [15].

Left ventricular global longitudinal strain (LV-GLS) was analyzed by a cardiologist, blinded to study data, using the Qlab13 (Philips Healthcare, Andover, Massachusetts) program (Fig. 2). While the end-diastole is considered the peak $\mathrm{R}$ wave of the electrocardiogram, end-systole was considered aortic valve closure. Mean GLS was calculated by averaging the peak GLS values of apical two-chamber, apical threechamber, and apical four-chamber images. Automatic endocardial margins were observed at the end-systole. Manual adjustments were made to ensure accurate tracking where necessary and to include LV wall thickness. The standard GLS limit was identified as $<-18 \%$ [16].

\section{Definitions}

The upper limit of the troponin test kit was $11.6 \mathrm{ng} / \mathrm{L}$ in our hospital laboratory. By taking the upper limit of troponin as reference, the patients were divided into two groups according to their hs-TnI levels at hospitalization. The standard GLS limit was identified as <-18\% [16]. 
Fig. 2 An example of global longitudinal strain speckle tracking apical four chamber view of myocardial injury patient



\section{Statistical analysis}

The Statistical Program for Social Sciences (for windows 20; SPSS Inc, Chicago, Illinois) was used for all statistical calculations. Continuous variables were tested for normal distribution using the Kolmogorov- Smirnov test. All continuous variables were represented as mean + standard deviation. Categorical variables were defined as a percentage. Since LVGLS, hemoglobin, creatinine, and white blood cell (WBC) were non-normally distributed Mann-Whitney $U$ test was used to compare between groups. The rest of continuous variables were normally distributed, and the Student t-test was used for the univariate analysis. The Chi-Square test was used to determine differences between groups for the categorical variables. For multivariate analysis, possible factors identified by univariate analyzes were assayed by logistic regression to identify independent predictors of abnormal LVGLS. A p value of less than 0.05 was considered statistically significant. Statistical analyses were performed using the SPSS software (Version 25.0, SPSS, Inc., Chicago, IL, institutionally registered software) Fig. 3.

\section{Results}

The study included 127 consecutive hospitalized patients diagnosed with COVID-19, and continued with 74 patients who remained after the exclusion criteria were applied. Echocardiographic imaging of the patients was performed $29.5 \pm 4.5$ days after discharge. Of the 74 patients, 45 were female (60.8\%), 29 were male (39.2\%). Patients' mean age was $59.9 \pm 11.85$; it was $66.4 \pm 12.3$ for the group with myocardial injury and $55.9 \pm 9.7$ for the group without myocardial injury $(\mathrm{p}<0.001)$. There were $18(64.3 \%)$ hypertensive patients in the group with myocardial injury, whereas there were $14(30.4 \%)$ hypertensive patients in the group without myocardial injury $(p=0.004)$. There was no significant

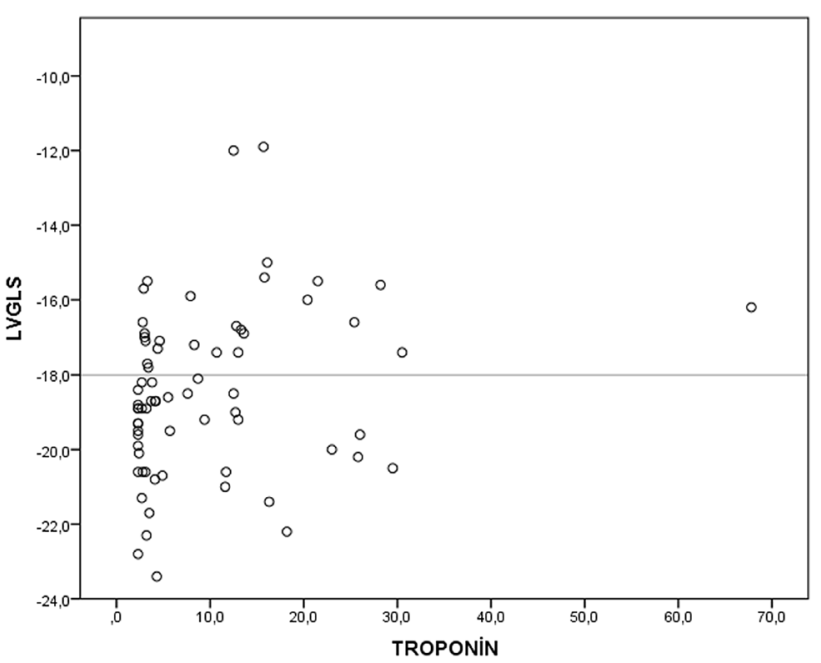

Fig. 3 Scatter plot graphic revealed the relation between troponin and LV-GLS values

difference between the groups in terms of diabetes mellitus $(\mathrm{p}=0.218)$ and smoking $(\mathrm{p}=0.265)$. Serum glucose $(\mathrm{p}=0.049), \operatorname{CRP}(74.7 \pm 94.2$ vs. $24.6 \pm 41, \mathrm{p}=0.02)$, serum troponin ( $62.9 \pm 126$ vs. $4.1 \pm 2.8, \mathrm{p}=0.02)$, D-dimer $(554.4 \pm 548$ vs. $218.1 \pm 204.4 \mathrm{p}=0.01)$, WBC $(8.5 \pm 4.4$ vs. $6.4 \pm 2.7, \mathrm{p}=0.014)$, and neutrophil levels $(6.4 \pm 4.4$ vs. $4.1 \pm 2.5, \mathrm{p}=0.07$ ) were higher in the group with myocardial injury than in the group without myocardial injury. There was no significant difference between the groups in serum creatinine, hemoglobin, platelet, and lymphocyte levels (All $p$ values $>0.05$ ) (Table 1$)$.

LV-GLS was found above -18 in $28(37.8 \%)$ patients. Sixteen $(57.1 \%)$ of these patients were in the group with the myocardial injury, and $12(26.1 \%)$ were in the group without myocardial injury $(\mathrm{p}=0.014)$. The average LVGLS values of the group with myocardial injury tended to be higher than the group without myocardial injury 
Table 1 Demographic and laboratory data of patients

\begin{tabular}{|c|c|c|c|c|}
\hline Variables & All patients $(n=74)$ & $\begin{array}{l}\text { Myocardial injury } \\
\text { presence }(\mathrm{n}=28)\end{array}$ & $\begin{array}{l}\text { Myocardial injury } \\
\text { absence }(n=46)\end{array}$ & $\mathrm{p}$ value \\
\hline Age & $59.9 \pm 11.8$ & $66.4 \pm 12.3$ & $55.9 \pm 9.7$ & $<0.001$ \\
\hline Female gender, n \% & $45(60.8)$ & $21(75)$ & $24(52.2)$ & 0.051 \\
\hline Hypertension n $\%$ & $32(43.2)$ & $18(64.3)$ & $14(30.4)$ & 0.004 \\
\hline Diabetes mellitus, $\mathrm{n} \%$ & $8(10.8)$ & $5(17.9)$ & $3(6.3)$ & 0.128 \\
\hline Smoking, n \% & $6(8.1)$ & $1(3.6)$ & $5(10.9)$ & 0.265 \\
\hline ASA, n \% & $4(5.4)$ & $3(10.7)$ & $1(2.2)$ & 0.115 \\
\hline ACE inhibitor, n \% & 13 (17.6) & $8(28.6)$ & $5(10.9)$ & 0.052 \\
\hline $\mathrm{ARB}, \mathrm{n} \%$ & $11(14.9)$ & $5(17.9)$ & $6(13)$ & 0.572 \\
\hline Diuretics, n \% & $19(25.7)$ & $11(39.3)$ & $8(17.4)$ & 0.037 \\
\hline Beta blocker, n \% & $8(10.8)$ & $6(21.4)$ & $2(4.3)$ & 0.022 \\
\hline $\mathrm{CCB}, \mathrm{n} \%$ & $10(13.5)$ & $6(21.4)$ & $4(8.7)$ & 0.120 \\
\hline Glucose, mg/dl & $129.5 \pm 48.6$ & $143.8 \pm 62.3$ & $120.8 \pm 36.2$ & 0.049 \\
\hline Creatinine, mg/dl & $0.87 \pm 0.24$ & $0.9 \pm 0.2$ & $0.85 \pm 0.2$ & 0.280 \\
\hline CRP, mg/l & $43.5 \pm 70.1$ & $74.7 \pm 94.2$ & $24.6 \pm 41$ & 0.02 \\
\hline Troponin, ng/l & $26.4 \pm 81.9$ & $62.9 \pm 126$ & $4.1 \pm 2.8$ & 0.02 \\
\hline D-dimer, mg/dl & $351.7 \pm 411.1$ & $554.4 \pm 548$ & $218.1 \pm 204.4$ & 0.01 \\
\hline $\mathrm{WBC}, \times 10^{3} / \mu \mathrm{L}$ & $7.2 \pm 3.6$ & $8.5 \pm 4.4$ & $6.4 \pm 2.7$ & 0.014 \\
\hline $\mathrm{Hb}, \mathrm{g} / \mathrm{dl}$ & $13.5 \pm 1.7$ & $13.1 \pm 1.3$ & $13.6 \pm 1.8$ & 0.192 \\
\hline $\mathrm{Plt}, \times 10^{3} / \mu \mathrm{L}$ & $215.1 \pm 75.4$ & $232.6 \pm 86.2$ & $204.9 \pm 67.2$ & 0.337 \\
\hline Neutrophyl, $\times 10^{3} / \mu \mathrm{L}$ & $4.9 \pm 3.5$ & $6.4 \pm 4.4$ & $4.1 \pm 2.5$ & 0.07 \\
\hline Lymphocyte, $\times 10^{3} / \mu \mathrm{L}$ & $1.5 \pm 0.66$ & $1.4 \pm 0.69$ & $1.5 \pm 0.65$ & 0.302 \\
\hline
\end{tabular}

$A S A$ acetylsalicylic acid, $A C E$ angiotensin converting enzyme, $A R B$ angiotensin receptor blocker, $C C B$ calcium channel blocker, $C R P C$ reactive protein, $W B C$ white blood cell, $H b$ hemoglobulin, Plt platelet
$(-17.7 \pm 2.6$ vs. $-18.9 \pm 1.8, \mathrm{p}=0.051)$. Also troponin levels were correlated with LV-GLS values $(r=0.22, p=0.045)$. Scatter plot graphic showed the relation between LV-GLS and troponin (Fig. 2). Left ventricular ejection fraction was lower in the group with myocardial injury $(58.9 \pm 2.1$ vs. $59.9 \pm 1.7, \mathrm{p}=0.032)$. Left atrium diameter $(36.1 \pm 5.3$ vs. $32.3 \pm 4.3, p=0.013)$, interventricular septum thickness $(11.5 \pm 1.9$ vs. $10.5 \pm 1.6, p=0.012)$, and left ventricular posterior wall thickness $(10.3 \pm 1.4$ vs. $9.4 \pm 1.4$, $\mathrm{p}=0.09$ ) were higher in the group with myocardial injury. There was no difference between the groups in terms of left ventricular end-systolic diameter, left ventricular enddiastolic diameter, left ventricular end-systolic volume, and left ventricular end-diastolic volume (Table 2).

LV-GLS was also correlated with CRP levels $(r=0.39$, $\mathrm{p}<0.001)$. In patients with abnormal LV-GLS $(>-18)$ had increased rate of higher CRP group (divided into two subgroups: $\geq 5 \mathrm{mg} / \mathrm{l}$ and $<5 \mathrm{mg} / \mathrm{l})(51.1$ vs. $96.6 \%, \mathrm{p}<0.001)$. Abnormal LV-GLS group was also related with LV diastolic diameters $(44.8 \pm 3.6$ vs. $46.9 \pm 4.1, p=0.029), \mathrm{LV}$ systolic diameters $(28.7 \pm 3.3$ vs. $30.7 \pm 4.6, p=0.035)$ and neutrophil count $(4.3 \pm 2.7$ vs. $6.1 \pm 4.4, \mathrm{p}=0.039)$. Other demographic, laboratory and echocardiographic data had no relation with abnormal LV-GLS. Only higher CRP level
(>5 mg/l) was found to be an independent predictor of abnormal LV-GLS (odds ratio $=6.5, \mathrm{p}=0.01$ ) $($ Table 3 ).

Electrocardiograms taken in the first month after the patients' discharge revealed T wave inversion in nine (12.2\%) patients and bundle branch block in two patients (one left bundle branch block, one right bundle branch block). There was no abnormality of PR and QT intervals or in the QTc value. There was no difference between the groups in PR and RR intervals and QRS, QT, and QTc durations (Table 2).

\section{Discussion}

Our study provides information about the electrocardiographic and echocardiographic evaluations of patients hospitalized for COVID-19 that had been made on first month follow-up after discharge. In echocardiography performed one month after discharge, $37.8 \%$ of all patients, $57.1 \%$ of the group with myocardial injury, and $26.1 \%$ of the group without myocardial injury revealed deterioration in LV-GLS values. This result gives rise to the thought that COVID-19 infection causes a high subclinical myocardial deformation rate in short-term follow-up. Mean LV-GLS values tended 
Table 2 Electrocardiography and echocardiography data of patients

\begin{tabular}{lcccc}
\hline Variables & All patients $(\mathrm{n}=74)$ & $\begin{array}{c}\text { Myocardial injury } \\
\text { presence }(\mathrm{n}=28)\end{array}$ & $\begin{array}{c}\text { Myocardial injury } \\
\text { absence }(\mathrm{n}=46)\end{array}$ & p value \\
\hline LVSD, mm & $29.5 \pm 3.9$ & $29.6 \pm 4.4$ & $29.4 \pm 3.7$ & 0.871 \\
LVDD, mm & $45.6 \pm 4.1$ & $46 \pm 4.7$ & $45.3 \pm 3.7$ & 0.531 \\
ESV, ml & $30.8 \pm 12.1$ & $33.8 \pm 14.7$ & $29 \pm 10$ & 0.297 \\
EDV, ml & $67.9 \pm 22.5$ & $70.3 \pm 23.8$ & $66.4 \pm 21.8$ & 0.644 \\
IVS, mm & $10.9 \pm 1.8$ & $11.5 \pm 1.9$ & $10.5 \pm 1.6$ & 0.012 \\
PW, mm & $9.8 \pm 1.4$ & $10.3 \pm 1.4$ & $9.4 \pm 1.4$ & 0.09 \\
LA, mm & $34.1 \pm 4.9$ & $36.1 \pm 5.3$ & $32.3 \pm 4.3$ & $\mathbf{0 . 0 1 3}$ \\
EF \% & $59.5 \pm 1.9$ & $58.9 \pm 2.1$ & $59.9 \pm 1.7$ & $\mathbf{0 . 0 3 2}$ \\
LVGS & $-18.5 \pm 2.2$ & $-17.7 \pm 2.6$ & $-18.9 \pm 1.8$ & 0.051 \\
PR interval, msec & $160.7 \pm 24.5$ & $159.5 \pm 23.7$ & $161.4 \pm 25.1$ & 0.485 \\
Heart rate, bpm & $73.7 \pm 11.9$ & $76.3 \pm 11.9$ & $72 \pm 11.8$ & 0.349 \\
QRS duration, msec & $91.6 \pm 13.9$ & $93.8 \pm 19.1$ & $90.2 \pm 9.7$ & 0.057 \\
QT duration, msec & $381.9 \pm 29.5$ & $386.9 \pm 27.3$ & $378.9 \pm 30.6$ & 0.499 \\
QTc duration, msec & $406 \pm 21.4$ & $415.3 \pm 20.4$ & $400.4 \pm 20.2$ & 0.973 \\
\hline
\end{tabular}

$E D V$ end-diastolic volume, $E F$ ejection fraction, $E S V$ end-systolic volume, $I V S$ interventricular septum, $L A$ left atrium, $L V G S$ left ventricular global longitudinal strain, $L V D D$ left ventricular end-systolic diameter, $L V S D$ left ventricular end-diastolic diameter, $P W$ posterior wall
Table 3 Independent predictors of LV-GLS (>-18)

\begin{tabular}{lll}
\hline Variables & Odds Ratio, 95 CI\% & p value \\
\hline CRP $(\geq 5 \mathrm{mg} / \mathrm{l})$ & $6.548(1.951-14.366)$ & $\mathbf{0 . 0 1 0}$ \\
LVSD, mm & $1.091(0.902-1.320)$ & 0.369 \\
LVDD, mm & $1.066(0.880-1.291)$ & 0.515 \\
Neutrophyl & $1.082(0.906-1.293)$ & 0.385 \\
\hline
\end{tabular}

$C R P \mathrm{C}$ reactive protein, $L V S D$ left ventricular end-diastolic diameter, $L V D D$ left ventricular end-systolic diameter

to be lower in the group with myocardial injury than in the group without myocardial injury.

Speckle tracking echocardiography is a better method for evaluating regional and global myocardial deformation than 2D echocardiography because it is independent of angle and does not require a fixed angle of insonation [17, 18]. Impaired LV-GLS is associated with worse cardiovascular outcomes in patients with preserved LVEF $[19,20]$. In the current study, degradation in LV-GLS was found in 37.8\% of the patients in their first month follow-up after COVID-19 infection. These results suggest that patients after COVID19 infection should be followed in terms of poor cardiovascular outcomes.

A meta-analysis evaluating 13 studies emphasized that increased troponin and D-Dimer levels in patients with COVID-19 were associated with high morbidity and mortality [21]. Elevated troponin levels are frequently observed during the hospitalization of patients with COVID-19, and this increase is associated with myocardial injury and mortality [21, 22]. Recent studies have reported that troponin's elevation in COVID-19 patients is associated with deterioration in LV-GLS levels [23]. The reduction in LVGLS from COVID-19 infection may be due to various factors. Viral infiltration of the myocardium can lead to cardiomyocyte death and inflammation [1,24]. Respiratory failure and hypoxia may also cause myocardial injury [25, 26]. Immune mechanisms that cause myocardial inflammation may occur with the immune response's activation and lead to the release of proinflammatory cytokines and adaptive immune response [25, 27]. Microvascular dysfunction, coronary plaque rupture, and thrombosis can also cause myocardial injury [28]. In the present study, even though $26.1 \%$ of the LV-GLS deteriorated in patients without myocardial injury; this rate increased to $57.1 \%$ in patients with myocardial injury. We found that hsCRP, an inflammatory marker that is frequently used in clinical practice, was an independent predictor of impaired LVGLS values in COVID-19 patients at 1 st month follow-up visits. This result suggests that LVGLS may deteriorate as a result of myocardial inflammation due to COVID-19.

In the active phase of the COVID-19 infection, electrocorticography anomalies such as premature atrial contraction, right bundle branch block (RBBB), $\mathrm{T}$ wave inversion, intraventricular block, and nonspecific repolarization have been reported and these anomalies are associated with increased mortality [29]. One month after recovered from COVID, T wave inversion was observed in 9 patients in the current study, and it was the most frequently observed ECG abnormality.

According to the latest American Echocardiography Association guidelines, for LVGLS, above $-18 \%$ is defined as normal, $-16 \%-18 \%$ as gray zone, under $-16 \%$ as diminished. [14]. In our study, we accepted the patient population 
staying in the gray zone as impaired LVGLS. We think that patients with LVGLS under -18\% should be followed closely in terms of cardiac involvement after COVID-19 infection.

There is no enough data in the literature about the risks encountered after the active period of COVID-19 infection and the approach to patient follow-up and treatment. Up to $100 \%$ of LV-GLS disruption has been reported in the active phase of COVID-19 infection [23]. In the current study, we observed subclinical cardiac deformation in one out of every three patients after COVID-19 infection, and this rate was higher in the group with myocardial injury. The fact that $26.1 \%$ of LV-GLS disorder was observed in the group without myocardial injury indicates that all patients should be evaluated in follow-up period after discharge, regardless of hospitalization troponin levels.

\section{Limitation}

The study was a single-center, and the number of patients included in the study was relatively small. There was no data on whether patients with myocardial deformation had any deformation before COVID-19 infection. In the present study, LVGLS rates were obtained at the 1st month followup after COVID-19 infection. Lack of control data regarding LVGLS values in patients with similar demographic characteristics without COVID-19 infection is one of the limitations of the study. In addition, excluding patient groups with increased troponin levels and deterioration in LVGLS values restricts the reflection of study results to large populations. However, the regression analysis reveals that LV-GLS is independently associated with CRP (cut of value five $\mathrm{mg} / \mathrm{l}$ and above) level, which is an indicator of inflammation in COVID-19 patients. We cannot exclude the possible impact of this lack of data on study results.

\section{Conclusion}

Myocardial LV-GLS values were impaired in one out of every three patients recovered from COVID-19 infection. This rate was $57.1 \%$ in the patient group with myocardial injury accompanying high troponin levels during the hospitalization period. Since impaired LV-GLS shows subclinical myocardial deformation and is independently associated with cardiac mortality, patients after COVID-19 infection may need to be followed closely in terms of clinical cardiac involvement.

Supplementary Information The online version contains supplementary material available at https://doi.org/10.1007/s10554-021-02211-5.

Funding None.

\section{Compliance with ethical standards}

Conflict of interest All the authors declared that they have no confict of interest.

\section{References}

1. Akbarshakh A, Marban E (2020) COVID-19 and the heart. Circ Res 126(10):1443-1455

2. Guan W-J, Ni Z-Y, Hu Y et al (2020) Clinical characteristics of coronavirus disease 2019 in China. New Engl J Med. https://doi. org/10.1056/NEJMoa2002032

3. Shi S, Qin M, Shen B et al (2020) Association of Cardiac Injury With Mortality in Hospitalized Patients With COVID-19 in Wuhan. China, JAMA Cardiol

4. Janus SE, Hajjari J, Karnib M, et al (2020) Prognostic value of left ventricular global longitudinal strain in COVID-19. Am J Cardiol

5. Li Y, Li H, Zhu S, et al (2020) Prognostic value of right ventricular longitudinal strain in patients with COVID-19. JACC Cardiovasc Imaging

6. Ruan Q, Yang K, Wang W et al (2020) Clinical predictors of mortality due to COVID-19 based on an analysis of data of 150 patients from Wuhan. China Intensive Care Med 46:846-848

7. Huang L, Zhao P, Tang D, et al (2020) Cardiac involvement in patients recovered from COVID-2019 identified using magnetic resonance imaging. JACC Cardiovasc Imaging

8. Stobe S, Richter S, Seige M, et al (2020) Echocardiographic characteristics of patients with SARS-CoV-2 infection. Clin Res Cardiol

9. Guo T, Fan Y, Chen M (2020) Chen Cardiovascular implications of fatal outcomes of patients with coronavirus disease 2019 (COVID-19). JAMA Cardiol. https://doi.org/10.1001/jamacardio .2020 .1017

10. Mehra MR, Desai SS, Kuy S, et al (2020) Cardiovascular disease, drug therapy, andmortalityin Covid-19. N Engl J Med

11. Carluccio E, Biagioli P, Alunni G et al (2018) Prognostic value of right ventricular dysfunction in heart failure with reduced ejection fraction: superiority of longitudinal strain over tricuspid annular plane systolic excursion. Circ Cardiovasc Imaging 11:e006894

12. Xie M, Li Y, Cheng TO et al (2015) The efect of right ventricular myocardial remodeling on ventricular function as assessed by twodimensional speckle tracking echocardiography in patients with tetralogy of Fallot: a single center experience from China. Int J Cardiol 178:300

13. Lyngbakken MN, Aagaard EN, Kvisvik B et al (2020) Cardiac troponin I and $\mathrm{T}$ are associated with left ventricular function and structure: data from the akershus cardiac examination 1950 study. Clin Chem 66:567-578

14. Lang RM, Badano LP, Mor-Avi V et al (2015) Recommendations for cardiac chamber quantifcation by echocardiography in adults: an update from the American Society of Echocardiography and the European Association of Cardiovascular Imaging. J Am Soc Echocardiogr 28:1-39

15. Schiller NB, Acquatella H, Ports TA et al (1979) Left ventricular volume from paired biplane twodimensional echocardiography. Circulation 60(3):547-555

16. Marwick TH, Leano RL, Brown J et al (2009) Myocardial strain measurement with 2-dimensional speckle-tracking echocardiography: defnition of normal range. JACC Cardiovasc Imaging 2:80-84

17. Ancona R, Comenale Pinto S, Caso P et al (2014) Left atrium by echocardiography in clinical practice: from conventional methods to new echocardiographic techniques. Sci World J 2014:451042 
18. Kalam K, Otahal P, Marwick TH (2014) Prognostic implications of global LV dysfunction: a systematic review and metaanalysis of global longitudinal strain and ejection fraction. Heart 100:1673-1680

19. Shah AM, Claggett B, Sweitzer NK et al (2015) Prognostic importance of impaired systolic function in heart failure with preserved ejection fraction and the impact of spironolactone. Circulation 132:402-414

20. Donal E, Lund LH, Oger E et al (2017) Importance of combined left atrial size and estimated pulmonary pressure for clinical outcome in patients presenting with heart failure with preserved ejection fraction. Eur Heart J Cardiovasc Imaging 18:629-635

21. Zheng Z, Peng F, Xu B, et al (2020) Risk factors of critical \& mortal COVID-19 cases: a systematic literature review and metaanalysis. J Infect

22. Tersalvi G, Vicenzi M, Calabretta D, et al (2020) Elevated troponin in patients with coronavirus disease 2019 (COVID-19): possible mechanisms. J Cardiac Failure

23. Baycan OF, Barman HA, Atici A, et al (2020) Evaluation of biventricular function in patients with COVID-19 using speckle tracking echocardiography. Int J Cardiovasc Imaging 1-10.

24. The European Society for Cardiology. ESC guidance for the diagnosis and management of CV disease during the COVID-19 pandemic. https://www.escardio.org/Education/COVID-19-andCardiology/ESCCOVID-19-Guidance.

25. Chen C, Zhou Y, Wang DW (2020) SARS-CoV-2: a potential novel etiology of fulminant myocarditis. Herz 45(3):230-232

26. Inciardi RM, Lupi L, Zaccone G et al (2020) Cardiac involvement in a patient with coronavirus disease 2019 (COVID-19). JAMA Cardiol. https://doi.org/10.1001/jamacardio.2020.1096

27. Madjid M, Safavi-Naeini P, Solomon SD et al (2020) Potential effects of coronaviruses on the cardiovascular system: a review. JAMA Cardiol. https://doi.org/10.1001/jamacardio.2020.1286

28. Varga Z, Flammer AJ, Steiger P et al (2020) Endothelial cell infection and endotheliitis in COVID-19. Lancet Lond Engl 395:1417-1418

29. McCullough SA, Goyal P, Krishnan U, et al (2020) Electrocardiographic findings in COVID-19: insights on mortality and underlying myocardial processes. J Cardiac Failure

Publisher's Note Springer Nature remains neutral with regard to jurisdictional claims in published maps and institutional affiliations. 